\title{
APPLICATIONS OF THE TRANSMITTED KIRCHHOFF-HOLMHOLTZ METHOD TO TRANSMITTED BODY WAVES AND POSSIBLE STRUCTURAL EFFECTS AT NTS
}

\author{
By Patricia Scott and Donald V. Helmberger
}

\begin{abstract}
We extend the Kirchhoff-Helmholtz integral method to calculate acoustic potentials which transmit through three-dimensional warped boundaries. We specify the potentials on an arbitrary surface with Snell's law and plane-wave transmission coefficients and numerically integrate their contributions at a receiver via the scalar integral representation theorem. The method is appropriate for modeling precritical transmitted potentials. Results from test models compare well with optical solutions for transmissions through a flat interface. We model the effect of several idealized crust-mantle boundary structures on teleseismic $P$ wave generated by explosion sources. The structures are all upwarps and are designed to produce travel-time residuals as a function of delta and azimuth which have the same magnitude as residuals observed for NTS tests within Pahute Mesa. These structures consistently cause complicated low amplitude waveforms which arrive early and simple high amplitude waveforms which arrive late. Thus, they cause systematic amplitude variations with azimuth, delta, and source location. The magnitude of this variation is less than or equal to $2 \frac{1}{2}$. This factor is somewhat less than the observed ab amplitude variation with azimuth of Pahute Mesa tests; however, it is approximately the same magnitude as the observed ab variation at a given station as a function of test location within the mesa.
\end{abstract}

\section{INTRODUCTION}

Despite dramatic improvements in the level of sophistication of data analysis, seismologists still cannot deterministically predict many observed amplitude and travel-time anomalies of body waves. One hypothesis to explain these anomalies is the presence of nonplanar velocity discontinuities such as sedimentary basins, mountains, and faults near the source or receiver. In order for us to assess the importance of this hypothesis, we need a technique for predicting the impact of near source and/or receiver structural complexity on far-field waves. In this paper, we present such a method based on the numerical evaluation of the KirchhoffHelmholtz integral with use of modified tangent plane boundary conditions. This method calculates the response of a wave which is transmitted through a warped boundary between two acoustic media. It contrasts from Scott and Helmberger (1983) where the reflections from a warped boundary are calculated. In this paper, we briefly describe the formalism of Kirchhoff-Helmholtz method for the transmitted case. Then, as an example of the method, we model the observed azimuthal amplitude and travel-time anomalies of short period $P$ waves from NTS blasts as a result of a geologic structure at the Moho.

\section{Formalism}

The method in this work is based on the numerical evaluation of the KirchhoffHelmholtz integral equation. The formalism differs slightly from that presented in Scott and Helmberger (1983). In that paper, we discussed the reflected wave solution; 
however, here, we state the Kirchhoff-Helmholtz solution for a transmitted potential. We also qualitatively discuss the assumptions involved in its use.

We wish to calculate a transmitted potential $\varphi_{2}$ at point $\epsilon$ in a homogeneous body $V_{2}$ resulting from an incident source potential located at point $x_{0}$ in a homogeneous body $V_{1}$. The boundary between the two bodies $\partial V$. The sound speeds and densities of $V_{1}$ and $V_{2}$ are $\alpha_{1}$ and $\alpha_{2}$ and $\rho_{1}$ and $\rho_{2}$, respectively. From the scalar integral representation theorem, we write the solution for $\varphi_{2}$ at a point $\underline{\epsilon}$ off the boundary


FIG. 1. (a) The geometry of the Kirchhoff-Helmholtz calculations for transmission across two acoustic media with sound speeds $\alpha_{1}$ and $\alpha_{2}$ and densities $\rho_{1}$ and $\rho_{2}$. The source is in $V_{1}$ at $\underline{x}_{0}$ and the receiver is in $V_{2}$ at $\underline{\epsilon}$. (b) A close up of a piece of the boundary which displays the angles.

$\partial V$, with $V_{2}$, at a time $t$ as

$$
\varphi_{2}(\underline{\epsilon}, t)=\int_{\partial v}\left[G_{2}(\underline{x}, \underline{\epsilon}, t)^{*} \underline{\nabla \Phi}(\underline{x}, t)-\Phi(\underline{x}, t)^{*} \underline{\nabla} G_{2}(\underline{x}, \underline{\epsilon}, t)\right] \cdot \underline{n_{2}}(\underline{x}) d S .
$$

Here ${ }^{*}$ denotes convolution and $\cdot$ denotes a vector dot product. $G_{2}$ is the fundamental singular solution of the scalar wave equation

$$
\frac{1}{\alpha_{2}^{2}} \frac{\partial^{2} G_{2}}{\partial t^{2}}-\nabla^{2} G_{2}=\delta\left(t-t_{0}\right) \delta(\underline{\epsilon}-\underline{x})
$$

In addition $\underline{n}_{2}$ is the outward pointing normal of $V_{2} . \Phi$ and $\underline{\nabla \Phi} \cdot \underline{n_{2}}$ are the potential 
and its normal derivative on the surface $\partial V$ in $V_{2}$. We display the geometry in Figure 1a for this problem.

Equation (1) is exact for the initial conditions that $\varphi_{2}(\underline{\epsilon}, 0)$ and $\dot{\varphi}_{2}(\underline{\epsilon}, 0)$ equal zero throughout $V_{2}$. The derivation can be found in Mao and Pao (1971) or Stratton (1941). To obtain equation (1), one requires that $\partial V$ is a reasonably smooth surface.

We now specify $G_{2}$ and $\Phi$. For a homogeneous medium, it is sufficient to use

$$
G_{2}(\underline{x}, \underline{\epsilon}, t)=\frac{\delta\left(t-\tau_{2}\right)}{4 \pi|\underline{x}-\underline{\epsilon}|} ; \quad \tau_{2}=\frac{|\underline{x}-\underline{\epsilon}|}{\alpha_{2}}
$$

If the incident field in $V_{1}$ results from an isotropic point source at $\underline{x}_{0}$, then $\Phi$ is approximated by

$$
\Phi=\frac{T f\left(t-\tau_{1}\right)}{\left|\underline{x}-\underline{x}_{0}\right|} ; \quad \tau_{1}=\frac{\left|\underline{x}-\underline{x}_{0}\right|}{\alpha_{1}}
$$

Here, $T$ is the acoustic plane wave transmission coefficient for a flat interface and is dependent on the local incidence angle at each point. $f(t)$ is the time function of the incident source function. The function in equation (4) approximates $\Phi$ well if the incident source field is of sufficiently high frequency such that every point on the surface transmits the incident pulse as though there were an infinite plane tangent to the surface at that point. Then the amplitude and the phase on the surface can be described locally by plane wave transmission coefficients and Snell's law. The value of the potential at one point is independent of the values at other points. Hence, the contributions of diffractions and multiple scattering to the potential are neglected.

We now estimate the normal derivatives $\partial \Phi / \partial n_{2}$ and $\partial G_{2} / \partial n_{2}$

$$
\begin{aligned}
& \frac{\partial \Phi}{\partial n_{2}} \simeq \frac{-T \dot{f}\left(t-\tau_{1}\right)}{\left|\underline{x}-\underline{x}_{0}\right|} \frac{\partial \tau_{1}}{\partial n_{2}} \\
& \frac{\partial G_{2}}{\partial n_{2}} \simeq \frac{-\dot{\delta}\left(t-\tau_{2}\right)}{4 \pi|\underline{\epsilon}-\underline{x}|} \frac{\partial \tau_{2}}{\partial n_{2}}
\end{aligned}
$$

The dot over the functions in (5) and (6) signifies time derivative. We approximate the normal derivatives by assuming the amplitudes of $G_{2}$ and $\Phi$ vary slowly on the surface relative to the phase. Hence, we can discard the terms

$$
\frac{1}{4 \pi} \delta\left(t-\tau_{2}\right) \frac{\partial}{\partial n_{2}}\left(\frac{1}{|\underline{x}-\underline{\epsilon}|}\right) \text { and } f\left(t-\tau_{1}\right) \frac{\partial}{\partial n_{2}}\left(\frac{T}{\mid \underline{x}-\underline{x}_{0}}\right) .
$$

Substitution of equations (3), (4), (5) and (6) into equation (1) yields

$$
\varphi_{2}=\frac{1}{4 \pi} \int_{\partial v} \frac{T \dot{f}\left(t-\tau_{1}-\tau_{2}\right)}{r_{0} r}\left(\frac{\partial \tau_{2}}{\partial n_{2}}-\frac{\partial \tau_{1}}{\partial n_{2}}\right) d S
$$

where $r=|\underline{x}-\underline{\epsilon}|$, the distance from the surface to the receiver, and $\tau_{0}=\mid \underline{x}-$ $\underline{x}_{0} \mid$, the distance from the source to the surface. We note that the discarded parts of 
normal derivatives are proportional to $1 / r_{0} r^{2}$ and $1 / r r_{0}{ }^{2}$. For problems computed in this study, the distance from the source to the surface averages $40 \mathrm{~km}$, and the distance from the surface to the receiver averages $20,000 \mathrm{~km}$. The $1 / r_{0} r^{2}$ and $1 / r r_{0}{ }^{2}$ terms are $6.25 \times 10^{-13}$ and $3.1 \times 10^{-9}$, respectively, and are small relative to the term $1 / r_{0} r$ in equation (7), which is $1.25 \times 10^{-7}$. We therefore discard these terms with confidence.

To estimate $\partial \tau_{1} / \partial n_{2}$ and $\partial \tau_{2} / \partial n_{2}$, we recall that the gradient of the phase is parallel to the normal of the wave front and has units of slowness. Thus,

$$
\begin{aligned}
\frac{\partial \tau_{1}}{\partial n_{2}} & =\frac{\cos \Theta_{1}}{\alpha_{1}} \\
\frac{\partial \tau_{2}}{\partial n_{2}} & =\frac{\cos \Theta_{2}}{\alpha_{2}}
\end{aligned}
$$

where $\cos \Theta_{1}$ is the cosine of the angle between the normal to the refracted wave front and the normal to the surface $\underline{n}_{2}$. It is equal to

$$
\cos \Theta_{1}=\left(1-\frac{\alpha_{2}^{2}}{\alpha_{1}^{2}} \sin ^{2} \Theta i\right)^{1 / 2}
$$

where $\Theta_{i}$ is the local incident angle calculated by

$$
\cos \Theta_{i}=\frac{\left(\underline{x}-\underline{x}_{0}\right)}{r_{0}} \cdot \underline{n}_{2} .
$$

$\cos \Theta_{2}$ is the cosine of the angle between the normal $\underline{n}_{2}$ and a ray connecting the surface and the receiver. Thus,

$$
\cos \Theta_{2}=\frac{(\underline{x}-\underline{\epsilon})}{r} \cdot \underline{n}_{2} .
$$

Figure $1 \mathrm{~b}$ shows a detailed picture of these angles. Substitution of the cosine factors (10) and (12) yields

$$
\varphi_{2}=\frac{1}{4 \pi} \int_{\partial v} T \dot{f}\left(t-\tau_{1}-\tau_{2}\right)\left(\frac{\cos \Theta_{2}}{r_{0} r \alpha_{2}}-\frac{\cos \Theta_{1}}{r_{0} r \alpha_{1}}\right) d S .
$$

The method for the calculation of equation (13) is discussed in Scott and Helmberger (1983). We simply calculate the integral as a summation of single point evaluations of the integrand. This method of integration requires that the elements which comprise the surface be small in length compared to the incident source wavelength. As in Scott and Helmberger (1983), we obtain the numerical ramp response from this integration and convolve it with the analytical third derivative of a Haskell isotropic source. Thus, we obtain $\dot{\varphi}_{2}$, the time derivative of the potential.

These calculations are appropriate for precritical transmission in a linear acoustic media. We do not allow the transmission coefficient to be complex. When the 
incident angle $\Theta_{i}$ exceeds the critical angle, $\Phi$ equals zero. This boundary value is consistent with geometric ray theory, but is not a realistic shadowing function. Rather, we expect $\Phi$ and $\partial \Phi / \partial n_{2}$ to vary smoothly across the shadow boundary if the surface is reasonably smooth. However, we argue that, under the circumstances considered here, the postcritical incidence portions of the integral contribute to the summed response of the transmitted potential at times much later than the specular portions and, therefore, are unimportant.

As a check of the method, we compute the transmitted response of a wave propagating through a planar boundary separating two volumes of different sound speeds. We then compare the numerical maximum amplitude with that obtained from the following first motion formula

$$
\varphi_{t}=T L f\left(t-\frac{r_{0}}{\alpha_{1}}-\frac{r}{\alpha_{2}}\right) .
$$

Here, $L$ is the spreading coefficient (Langston, 1977; Hong, 1978)

$$
L=\left\{\left(\frac{z_{1}}{\eta_{v 1}}+\frac{z_{2}}{\eta_{v 2}}\right)\left(\frac{z_{1}}{v_{1}^{2} \eta_{v 1}}+\frac{z_{2} \eta_{v 1}^{2}}{v_{2}^{2} \eta_{v 2}^{3}}\right)\right\}^{1 / 2}
$$

where $z_{1}$ and $z_{2}$ are the vertical distances of the source and receiver, respectively, from the boundary. $\eta_{v i}$ is

$$
\eta_{v i}=\left(\frac{1}{\alpha_{i}^{2}}-p_{0}^{2}\right)^{1 / 2}
$$

where $p_{0}$ is the ray parameter.

Figure 2 shows examples of this comparison. We have computed transmitted potentials for an incident isotropic source which is the first derivative of a Haskell source with parameters $(B=2, K=10)$. The velocity and density model used for the comparison calculations are shown in the top of Figure 2b.

We show two Kirchhoff synthetics in Figure 2a to demonstrate the nature of truncation phases which can contaminate the synthetics. These phases arrive approximately $3 \frac{1}{2}$ sec after the first arrival in both synthetic A and synthetic B and they are artifacts of the technique. In synthetic $A$, the phase is a result of the finiteness of the grid. A diagram to the left of the synthetic shows this effect. The grid is a square with a length of $150 \mathrm{~km}$. The source is $500 \mathrm{~km}$ above the center of the grid and the receiver is $1000 \mathrm{~km}$ below the source. From this diagram, we observe that the edge interferes at a time $t_{2}=r_{02} / \alpha_{1}+r_{2} / \alpha_{2}$. The geometric ray arrives at a time $t_{1}=r_{01} / \alpha_{1}+r_{1} / \alpha_{2}$. Hence, the truncation phase arrives $3.6 \mathrm{sec}$ later than the first arrival in synthetic $A$.

In synthetic $B$, the phase is a result of the shadowing function. We use the same grid to calculate synthetic B as for synthetic A; however, the source is $167 \mathrm{~km}$ above the interface. For this velocity model, the local angle between the incident ray and the normal to the surface exceeds critical when the distance from the center of the grid exceeds $50 \mathrm{~km} . \Phi$ on the surface is 0 beyond this distance. This abrupt change in boundary conditions introduces a truncation phase into the synthetic. From the 
diagram to the left of synthetic, we see that this phase arrives $4 \mathrm{sec}$ later than the geometric arrival. The truncation phase in synthetic A caused by grid finiteness does not constitute a problem. If it contaminates the phase of interest, we can enlarge the grid appropriately. However, the truncation phase in synthetic B caused

a)


FiG. 2. (a) Two synthetics and the grid geometry used to compute them. Synthetic A is contaminated by a truncation phase which originates from the edge of the grid. Synthetic $B$ is contaminated by a phase which originates from the abrupt change in boundary conditions. The grid next to synthetic $B$ is gray when $\varphi=0$ on the boundary. (b) A comparison between Kirchhoff-Helmholtz and first motion solutions. The input source is the first derivative of a modified Haskell function with parameters $(B=2, K=10)$. The maximum dimensionless amplitude of the source input functon is 45.1 .

by the boundary conditions fundamentally restricts the source-receiver geometries we can investigate.

Figure 2b shows a profile of Kirchhoff synthetics for a source $500 \mathrm{~km}$ above the interface and five receivers $500 \mathrm{~km}$ below the interface. The horizontal distance, $x$, of the receivers ranges from $0 \mathrm{~km}$, directly underneath the source, to $755 \mathrm{~km}$. The two columns next to the synthetics contain the numerical peak amplitudes and the 
predicted amplitude from equation (15). The agreement is good. We cannot calculate a response past $x=755 \mathrm{~km}$ because a truncation phase resulting from the boundary conditions on the interface starts to interfere with the direct arrival. We must always take care to avoid such contamination.

\section{NTS StruCture (AN ExAMPLE OF NeAR-Source EFFECTS)}

We now apply the method by modeling the effects of idealized Moho structures on transmitted teleseismic $P$ waves generated by nuclear tests in Pahute Mesa, Nevada Test Site. We wish to ascertain whether focusing-defocusing by structure on the Moho explains the unusual behavior of amplitudes from these tests.

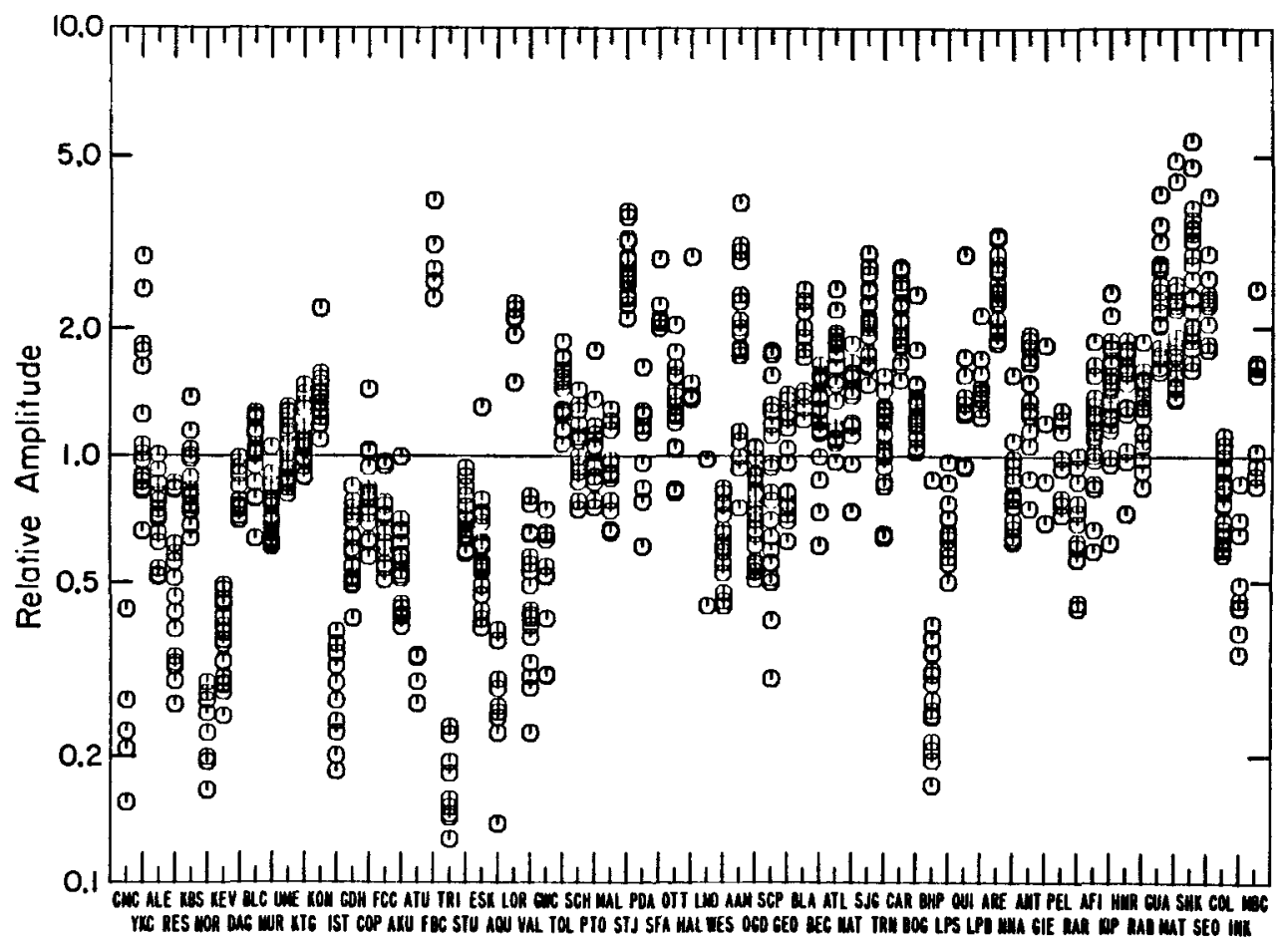

FIG. 3. The short-period $P$-wave $a b$ amplitude data set for 25 Pahute Mesa events plotted as a function of station location. The amplitudes are corrected for event size, geometric spreading, and instrument gain at $1 \mathrm{sec}$ and are plotted relative to a master event (from Lay et al. 1983a).

We review these anomalous observations of short-period $P$ waves from Pahute Mesa. Figure 3 is a plot of $1200 a b$ amplitude measurements from 25 tests within Pahute Mesa as a function of station location from Lay et al. (1983a). The $a b$ amplitudes are measured from the first peak to the first trough. They are corrected for geometric spreading, the instrument gain at $1 \mathrm{sec}$ and event size, following a procedure developed by Butler (1984). The amplitudes are relative to a master event which minimises the overall scatter of the data.

The data has two important features. First, the relative amplitudes range from 0.13 at station TRI to 5.1 at station SHK. This variation is nearly a factor of 40 . Most stations between the azimuths $0^{\circ}$ and $60^{\circ}$ have significantly lower amplitudes than those between $60^{\circ}$ and $120^{\circ}$. Second, the relative amplitudes at a given station vary by a factor of $2 \frac{1}{2}$ as a function of event location within the mesa. The latter 
variation clearly originates from a near-source mechanism because the events are separated by, at most, $15 \mathrm{~km}$.

If one calculates the mean relative amplitude at each station, then the overall amplitude variation with azimuth reduces to a factor of 12 (Lay et al., 1983a). The next two figures suggest that this large amplitude scatter is also caused by a nearsource mechanism. Figure 4, from Lay et al. (1983a), shows the azimuthal pattern of relative amplitudes for GREELEY, an event within the mesa, and FAULTLESS, an event $100 \mathrm{~km}$ outside the mesa. Although both events have comparable yields,


FIG. 4. The relative $a b$ amplitudes of GREELEY and FAULTLESS as a function of station location (from Lay et al., 1983a).

their azimuthal patterns differ substantially. This difference is particularly obvious between $0^{\circ}$ and $90^{\circ}$. Figure 5 displays plots from Lay et al. (1983b) which enhance the difference between patterns of events in the mesa and events outside the mesa. These plots are ratios of amplitudes of three events outside the mesa (FAULTLESS, PILEDRIVER, and BILBY) divided by the average mesa amplitudes. These ratios are an approximate measure of a near-source anomaly if the FAULTLESS, PILEDRIVER, and BILBY patterns are only influenced by path and receiver effects and are, therefore, constant as a function of azimuth. Furthermore, the path and receiver 
effects must be characterized by multiplicative factors. Because the ratio patterns for all three events are similar, these assumptions are probably true. Therefore, the factor of 13 variation of these ratios between $0^{\circ}$ and $120^{\circ}$ is roughly an estimate of the near-source anomaly at the mesa.

To see if this amplitude variation correlates with waveform changes, we plot in Figure 6 several seismograms at stations between $30^{\circ}$ and $100^{\circ}$ which recorded both FAULTLESS and GREELEY. The top and bottom seismograms are recordings of FAULTLESS and GREELEY, respectively, with their absolute $a b$ amplitudes in



FIG. 5. Ratios of relative $a b$ amplitudes of FAULTLESS, PILEDRIVER, and BILBY divided by the average relative $a b$ amplitudes of the mesa events (from Lay et al., 1983b).

millicrons, corrected for instrument gain only. There is no obvious waveform differences in the GREELEY records which correlate with the dramatic $a b$ amplitude changes. Furthermore, we do not see any obvious difference in frequency content and/or complexity between low stations and high stations for either event. However, there are some systematic differences between GREELEY and FAULTLESS seismograms. A shoulder occurs 2 to 3 sec after the first arrival on GREELEY records (e.g., STU, PTO, MAL, STJ, OTT, GEO, and ATL). Lay has also seen these arrivals for other mesa events (Lay et al., 1983b). No such arrival is apparent on the FAULTLESS seismograms. Also the width of the first pulse of GREELEY 
seismograms is narrower than those of FAULTLESS seismograms at a few stations (e.g., SJG, ATL, BLA, GEO, SCP, and STU). Both phenomena, though, occur throughout the azimuthal range and do not correlate with the $a b$ amplitude changes.

The data demonstrates that near-source anomalies cause a variation of $2 \frac{1}{2}$ of relative $a b$ amplitudes at a given station as a function of event location within the mesa. Moreover, near-source anomalies also cause part of the $a b$ amplitude variation with azimuth (or station location) from mesa events. We cannot completely eliminate contamination of the azimuthal pattern by path and near-receiver effects. Certainly near-receiver effects can be as large as those observed for the Pahute mesa tests (Butler, 1984). Yet the similarity of the ratio patterns of FAULTLESS,



FIG. 6. Seismograms from FAULTLESS (top record) and GREELEY (bottom record) displayed in order of increasing azimuth in the range of $30^{\circ}$ to $100^{\circ}$. Also shown are the absolute $a b$ amplitudes in millimicrons, corrected only for instrument gain at $1 \mathrm{sec}$.

BILBY, and PILEDRIVER suggests that the pattern for mesa tests, seen in Figure 3 , is dominated by a near-source mechanism. Finally, the variation of relative $a b$ amplitudes with azimuth does not correlate with any obvious waveform changes for a typical mesa event, GREELEY. There is no definitive evidence to determine whether $a b$ variations correlate with travel-time residuals.

In this paper, we assume that all the observed amplitude anomalies result from near-source mechanisms. We then test the hypothesis that structure on the Moho, consistent with travel-time residuals, focuses or defocuses $P$ waves enough to produce the magnitude of the amplitude anomaly. There are alternative near-source explanations for these anomalies. In addition, to the focusing-defocusing hypothesis, 
workers (Lay et al., 1983; Wallace et al., 1983) postulate that the movement of faults associated with nuclear blasts causes a superposition of distributed or point doublecouple sources with the isotropic bomb source. The amplitude anomalies are, then, the radiation pattern caused by a double-couple source. Longer period studies of Love/Rayleigh ratios, $P$ nl, $P$ and $S$ waves (Nuttli, 1969; Aki and Tsai, 1972; Wallace et al., 1983) generated by these blasts support the latter hypothesis. However, we speculate that, as the frequency content of the signal increases, the role of lateral near-source structure in distorting amplitudes becomes more important. From travel-time residual studies (Spence, 1974; Minster et al., 1981) workers have deduced that there is a high-velocity zone directly beneath the Silent Canyon Caldera in the mesa which extends down to $100 \mathrm{~km}$. Such a velocity structure may cause amplitudes which deviate from those predicted by a spherically symmetric Earth model.

To investigate how geology can affect amplitudes, we presume that the apparent velocity variations deduced from the travel-time residuals are a manifestation of Moho topography. We exclude from consideration the impact of the Silent Canyon volcanics on transmitted $P$ waves because both Spence and Minster correct the residuals statically for these low velocity rocks; thus the residual patterns are not a result of the caldera. In any case, we cannot readily model a feature so close to the source. If we place a strong velocity discontinuity, such as that between volcanic and granite rocks, closer than $10 \mathrm{~km}$ to the source, we generate a truncation phase which interferes with the transmitted $P$ phase.

We describe the Earth with a two layer crust-mantle velocity model. The velocity of the uper layer is $6.5 \mathrm{~km} / \mathrm{sec}$ and that of the lower is $8 \mathrm{~km} / \mathrm{sec}$. The depth of the interface is $45 \mathrm{~km}$. The receivers are located at distances such that the $1 / R$ amplitude decay corresponds to spreading at teleseismic distances between $60^{\circ}$ and $70^{\circ}$ for a JB Earth (Langston and Helmberger, 1975).

The number of ways to distort the Moho is infinite. We, therefore, restrict ourselves to a few three-dimensional topographies where the maximum height of the anomaly is $10 \mathrm{~km}$ and the maximum width is approximately $25 \mathrm{~km}$. The choice of these values is based on both the Spence (1974) and Minster et al. (1981) studies. They find an advance of $\approx 0.25$ to $0.4 \mathrm{sec}$ for nearly vertical rays from shots within the caldera. As these rays become shallow, this advance lessens or disappears completely. From crude calculations, we estimate that $10 \mathrm{~km}$ of upward relief on the Moho will produce the required timing anomalies of these rays. Furthermore, we confine the relief laterally so that rays exiting the mesa at shallow angles are unaffected by the structure. We recognize that these structures are extreme. However, if we cannot produce the observed amplitude anomalies with these topographies, we can discard structure on the Moho as the dominated cause of these anomalies.

Of the infinite number of structures, we arbitrarily select four examples with height $c=10 \mathrm{~km}$ and width $w=25 \mathrm{~km}$. These topographies are described by simple analytical formulas and are convenient to use. The topographies with their labels are as follows

$$
\text { Upwarp: } \begin{aligned}
Z & =Z_{c o n}+\frac{c}{2}(\cos (2 \pi((r-w / 2) / w))-1) \text { if } r \leqq \frac{w}{2} \\
Z & =Z_{\text {con }} \quad \text { if } r>\frac{w}{2}
\end{aligned}
$$




$$
Z=Z_{\text {con }}-c \text { if } r \leqq \frac{w}{2}
$$

Plug: $Z=Z_{\text {con }}+\frac{c}{2}\left(\cos \left(2 \pi\left(\left(r-\frac{w}{2}-5\right) \mid w\right)\right)-1\right)$ if $\frac{w}{2}<r \leqq \frac{w}{2}+5$

$$
Z=Z_{\text {con }} \quad \text { if } \quad r>\frac{w}{2}+5
$$

$$
\text { Sinc: } Z=Z_{c o n}-c \operatorname{sinc}\left[\frac{21.991 r}{w}\right] \text {. }
$$



FiG. 7. Transmitted potentials from sources $35 \mathrm{~km}$ above the center of the structure. The crosssections of the structures upwarp, exponential, plug, and sinc are above the synthetics. For comparison, potentials which propagate through a flat boundary are shown in the first column. The potentials are from receivers which are $20,000 \mathrm{~km}$ below the source and which vary from 0 to $7000 \mathrm{~km}$ horizontally away from the source. All amplitudes are multiplied by 0.01 .

Here, $r=\sqrt{\left(x-x_{c}\right)^{2}+\left(y-y_{c}\right)^{2}}$ and is the horizontal distance of each point on the surface from the midpoints of the grid $\left(x_{c}, y_{c}\right) . Z_{c o n}$ is the baseline level of the Moho and is $45 \mathrm{~km}$ for all the calculations. The values of the constants in the exponential and the sinc bumps confine the anomaly's width to approximately 25 $\mathrm{km}$. A schematic cross section of each topography is shown in Figure 7. All the structures are symmetric with azimuth.

Initially, an isotropic source is directly above the center of the structure; thus, the transmitted potential is only a function of $x$ and $z$. The source is $45 \mathrm{~km}$ above the baseline of the Moho. The transmitted potential is calculated at receivers which are $20,000 \mathrm{~km}$ below the aource. The horizontal distance of the receivers from the center of the topography ranges from 0 to $7,000 \mathrm{~km}$. Figure 7 shows the transmitted 
potential and the peak amplitude as a function of $x$ in increments of $1000 \mathrm{~km}$ for each of the four topographies. In addition, the responses for a wave which transmits through a planar boundary are displayed in the first column. The corresponding take-off angle for the flat boundary synthetics are to the left of the column. By comparing these synthetics with those in the other columns, we can determine how much distortion of the waveform is caused by each structure. The synthetics in Figures 7 and 10 do not include a $Q$ and instrument operator or a reflected $p P$ phase. Although these effects are important, we want to examine amplitude and waveform distortions caused by structure with a simple input pulse. The ringing caused by an instrument or $p P$ may mask the presence of multiple arrivals caused by the topography.

All the structures cause intriguing changes in the waveforms and arrival times of the synthetics. The waveform features originate from timing changes caused by each topography. Each point of the Kirchhoff synthetic originates from elements which are illuminated by the source and, in turn, illuminate the receiver at a total travel time, $\sigma=\tau_{1}+\tau_{2}$. We can associate, with each element of the grid, a value of total travel time, $\sigma(x, y)$. The total travel-time function on the surface depends on the source location, the receiver location, and the surface geometry. Figure 8 shows examples of this function. Here, we calculate $\sigma(x, y)$ for elements which make up a flat interface (Figure 8a), an upwarped interface (Figure 8b), a plug (Figure 8c) and a sinc function (Figure 8d). In each example, the source is directly above the center of the structure and is $45 \mathrm{~km}$ above the baseline of the interface. The receiver is $20,000 \mathrm{~km}$ directly below the source. The contours of constant total travel time are projected onto the topography (top figure in $8, a$ through $d$ ). We also display these contours as a function of $x$ and $y$ (bottom figure in $8, a$ through $d$ ). The contours are circles because of the particular source-receiver geometry. For the sake of brevity, we only show that portion of the grid which contributes to the initial second of each Kirchhoff synthetic. The synthetics which correspond to these total traveltime functions are also shown (middle figure in $8, a$ through $d$ ). These figures show how structure on the interface distorts the total travel-time contours and, as a result, produces multiple arrivals in the synthetic.

We examine this effect in detail. The contours are in intervals of $0.125 \mathrm{sec}$ as are the tick marks below the synthetics in Figure 8. The geometric arrival time occurs at the center of the contour plot. Thus, by counting the contours, we can estimate the cumulative area of the surface which contributes to the synthetic at a given time. We deduce, from Figure 8, that, approximately,

$$
\left.A(t) \alpha \frac{d S}{d t}\right|_{t} .
$$

$A(t)$ is the amplitude of the response at time $t . S$ is the total area of the surface which contributes to the response at time $t$. For example, the initial $0.375 \mathrm{sec}$ of the synthetic from a flat interface results from a rapid increase in the cumulative area of the surface which is illuminated between $t=0.125$ and $t=0.25 \mathrm{sec}$. After $t=$ $0.25 \mathrm{sec}$, the area of the surface is illuminated at a constant rate. Thus, the resultant synthetic can be viewed as a convolution of the source time function with a step function which starts between $t=0.125$ and $0.25 \mathrm{sec}$.

We quantify this statement by following an approach developed by Hilterman (1975) and Haddon and Buchen (1981). The symmetry of the source-receiver geometry and the surface geometry allows us to recast the integral (13) as a one- 




FIG. 8. Travel-time contours for a source $35 \mathrm{~km}$ directly above the structure and receivers $20,000 \mathrm{~km}$ directly below the source. The four structures are: (a) a plane; (b) an upwarp; (c) a plug; and (d) a sinc function. The contours are projected onto the topographies and flat grids. The synthetics which correspond to each travel-time projection are in between the two projections. The contour interval is $0.125 \mathrm{sec}$ as are the tick marks of the synthetics. The geometric arrival time is the center of the contours.

dimensional integration with respect to total travel time, $\sigma$. If the transmission coefficient varies slowly over the surface, then

$$
\varphi_{2} \cong \frac{T}{4 \pi} \dot{f}^{*}\left(\frac{\dot{\Omega}_{2}}{\alpha_{2}}-\frac{\dot{\Omega}_{1}}{\alpha_{2}}\right)
$$

where

$$
d \Omega_{1}=\frac{\cos \Theta_{1}}{r_{0} r} d S
$$


and

$$
d \Omega_{2}=\frac{\cos \Theta_{2}}{r_{0} r} d S
$$

$\dot{\Omega}_{1}$ and $\dot{\Omega}_{2}$ are the time derivatives of modified solid angles. $\Omega_{1}$ is a modified solid angle with vertex at the source subtended by the surface $S$, and $\Omega_{2}$ as a modified solid angle with vertex at the receiver subtended by $S$.

We now examine the origins of the multiple arrivals in the potentials which propagate through interfaces with structure. For example, the amplitude and frequency content of synthetics from the upwarp (column 3 of Figure 7) are controlled by the interference of two pulses. The travel-time contours in Figure $8 \mathrm{~b}$ for the upwarp differ considerably from those of a flat interface (Figure 8a). Far less of the upwarped surface is illuminated within $0.25 \mathrm{sec}$ of the geometric arrival time. Furthermore, the upwarp topography causes subtle changes of the width between travel-time contours. There are two locations where this change occurs: (1) at the top of the upwarp and (2) at the edge of the upwarp. The first pulse in this synthetic originates from the elements in the first location while the second pulse originates from the second location. Because the ring of elements which contribute to the second pulse has a larger area than that of elements which contribute to the first pulse, the second pulse is larger than the first pulse.

As the receivers move away from the center, the maximum amplitudes decrease as a result of the interference of the two pulses. We destroy the symmetry of the surface illumination by moving the receivers horizontally. The illumination of elements, which initially was simultaneous, now occurs at slightly different times and causes destructive interference; this destructive interference causes a reduction in peak amplitudes and the broadening of the pulse widths for both phases. Moreover, as the receiver moves out laterally, the planar part of the boundary becomes more important in controlling the amplitude of the transmitted pulse. Hence, the amplitudes, travel times, and the waveforms of distorted pulses approaches those of a pulse which has propagated through a flat boundary. This phenomena is present in almost all the top synthetics in Figure 7.

Other intriguing features are present in the synthetics shown in Figure 7 . The potentials transmitted through the sinc and exponential bumps shown in columns 3 and 5 have an apparent delay which is not seen in the other synthetics. These two topographies drop in height near the peak more rapidly than does the upwarp topography. Consequently, fewer elements are illuminated and contribute to the transmitted potential at times near the geometric arrival time. This is illustrated for the sinc topography in Figure 8d. Hence, the amplitude near the geometric arrival time is lower than amplitudes at later times.

The opposite is true for the synthetics of waves which are transmitted through a plug. They are displayed in column 4 of Figure 7. The topography and travel-time contours for the bottom synthetic from this column are shown in Figure 8c. This figure shows that more elements are illuminated and contribute to the response near the geometric arrival time for this topography than for the upwarp, sinc, and exponential topographies. The resultant synthetic is made up of two pulses of equal size. Each pulse has the amplitude and shape of a wave which has transmitted through a planar interface. The plug is essentially comprised of two planar interfaces, one at $z=45 \mathrm{~km}$ and the other at $z=35 \mathrm{~km}$. The edges of the plug have been tapered to avoid a shadowing problem. While the difference in the interface depths 
does not alter the amplitudes of the pulses, it does change the arrival times. This slight separation in arrival time causes the observed interference pattern. As the receiver moves out laterally, the timing between the two pulses changes. The pulse width of the first arrival narrows while the width of the second one broadens. By transmitting a wave through such a structure, we vary the maximum amplitude of the synthetics by a factor of $2 \frac{1}{2}$.

We confirm that these structures are approximately producing the correct traveltime anomalies. We plot the residuals, in addition to the peak amplitudes of the synthetics, as a function of distance to discern any systematic relationship between



FIG. 9. Plots of peak amplitudes, amplitudes of first pulses, and travel-time residuals as a function of distance from synthetics in Figure 7. The different symbols correspond to different topographies and are at the bottom of the figure. Where first pulse amplitudes are different from peak amplitudes, the values of first pulse amplitudes are plotted with open symbols and the peak amplitudes are plotted with closed symbols. The dotted line corresponds to the peak amplitudes from synthetics which propagate through a planar interface.

the two parameters. We also plot the amplitude of the first pulse if the synthetic is made up of multiple arrivals. This amplitude is measured from the start of the synthetic to the first peak. The plots are displayed in Figure 9. The travel-time "residuals" are defined by the difference between the arrival times of transmissions through a bumpy surface and the times of transmissions through a flat surface. Where there is an apparent delay in the synthetics such as in those from the exponential and sinc bumps, we measure the arrival time at the start of the upswing. The amplitudes are uncorrected for geometric spreading. The change of amplitude from spreading, seen in the synthetics in column 1, is negligible in the distance range of interest; hence no correction is necessary. 
The "residuals" in Figure 9 produced by these structures behave in a predictable fashion. The transmitted potentials which propagate vertically to stations between 0 to $4,000 \mathrm{~km}$ experience the most advance. The exceptions to this behavior are residuals from the sinc and exponential synthetics. We know there is some energy arriving at these nearly vertical stations with $0.3 \mathrm{sec}$ advance from the previous discussion. However, because the energy is so small relative to later pulses, these synthetics appear to have delays.

When the paths of the potentials become shallower, we see that the advance disappears. The planar part of the interface begins affecting the travel times and waveforms. The arrival times of the transmissions through the bumps approach those of transmissions through a flat interface. The exception to this pattern is the residuals of the plug synthetics. These synthetics have an advance of $0.3 \mathrm{sec}$ which is constant as a function of horizontal distance. The behavior results from the constant height of the plug across the entire width of the bump.

What is the relationship between the travel-time anomalies and the amplitude anomalies? We predict that as the magnitude of the travel-time anomalies decreases the magnitude of the amplitude anomalies decreases also. The amplitudes, as well as the arrival times, will be controlled by the planar part of the surface. This relationship is observable in Figure 9. The amplitudes, except in one case, start to approach the value of 0.002 at distances ranging from 5,000 to $7,000 \mathrm{~km}$. The exception is the maximum and first pulse amplitudes of the synthetics from the plug topography. These values appear to systematically decrease with distance. However, these values do approach the planar amplitudes at distances beyond 7000 $\mathrm{km}$.

Furthermore, waves which arrive earlier than is predicted by planar calculations also have lower amplitudes than is predicted. Contrarily, the synthetics from the sinc topography arrive late and have anomalously high amplitudes. Indeed, the pattern of residual variation is precisely mimicked by the pattern of amplitude variation for this structure. The mimicking of amplitude and travel-time anomalies also occurs for synthetics from the exponential bumps.

This mimicking does not occur for synthetics from the upwarp and plug topographies. Each of these synthetics consists of multiple arrivals. Thus, if we take the maximum amplitude as a measure of amplitude anomaly and compare with traveltime anomalies, we do not see an obvious correlation between the two parameters. The travel time is perturbed by a relatively small part of the surface. The maximum amplitude is perturbed by a much larger part of the surface. It is a less local property of the topography. If a broader band source time function interacts with the surface, the amplitude anomaly would change but not the travel time anomaly. To improve the correlation, we measure the amplitude of the first pulse of the synthetic if it is different from the maximum amplitude. These values are shown in Figure 9 by the open circles and triangles for the upwarp and plug synthetics. We do not improve the visual correlation significantly.

The modeling of a symmetric structure demonstrates that such a structure on the Moho, consistent with travel-time residuals, causes a factor $2 \frac{1}{2}$ in maximum amplitude variation as a function of distance. The variation of amplitudes of first pulses is somewhat less. Neithe $e_{\perp}$ variation is as large as the observations of amplitude changes between stations for a given test at NTS. Furthermore, where there is a large amplitude variation, there is significant waveform distortion of the synthetics. Also, the bumps generally cause low amplitudes. We note that the mesa data set has both anomalously high and low amplitudes. The low amplitude synthetics arrive 
early. However, any relationship between amplitude anomalies and travel-time anomalies is dependent on frequency because the effect of a structure on a wave is dependent on frequency.

The modeling to this point produces anomalies which are dependent only on distance because the structures are symmetric. We now introduce asymmetry into the problem by allowing the source to move off the center of a symmetric upwarp. We do these calculations because observed travel-time and amplitude anomalies are presented as a function of azimuth. Yet we do not know if these anomalies arise from azimuthal or delta heterogeneities. Additionally, there is an observed variation of amplitude at a given station with a change in source position in the mesa. If we change the source position across a sample structure, can we reproduce the factor of $2 \frac{1}{2}$ seen in Figure 3? We also wish to examine whether there is any systematic relationship between amplitude and travel-time anomalies as a function of azimuth as we have done previously with these parameters as a function of distance.

The modeling experiment is similar to the previous one. The receivers are 1000 to $7000 \mathrm{~km}$ horizontally away from the center of the topography and $20,000 \mathrm{~km}$ below the source. The sources are $45 \mathrm{~km}$ above the baseline of the Moho. To produce the azimuthal anomalies in the synthetics, we move the source off the center of the upwarp in one direction in increments of $2 \mathrm{~km}$. The responses are calculated for seven distances at five different azimuths. We select the topography upwarp for this experiment. The choice of topography is somewhat arbitrary; however, we make this particular choice because this topography causes substantial variation in amplitudes as a function of distance. If this topography fails to produce much azimuthal variation, then the other topographies will fail to do so, also.

Figure 10 shows our results. A crosssection of the source-receiver configuration and the geometry of the upwarp is in the center of the figure. In addition, a topographic map of the center portion of the grid is displayed. The contours are in kilometers, and the maximum height of the bump is $10 \mathrm{~km}$. The topography map also shows the source locations and the azimuthal lines along which the calculations are done. The resulting waveforms and maximum amplitudes surround these diagrams. Each group of 28 waveforms is calculated for the corresponding azimuth. The groups are made up of four columns of synthetics corresponding to calculations done with the source location designated at the top of each column. Sources A, B, $\mathrm{C}$, and $\mathrm{D}$ are, respectively, 2, 4, 6 and $8 \mathrm{~km}$ from the center. The rows correspond to calculations done at the horizontal distances next to the row. In Figures 11 to 13, we plot the travel-time residuals, peak amplitudes, and first pulse amplitudes obtained from these synthetics as a function of azimuth for each distance.

There is a change in overall complexity of the synthetics as a function of azimuth. The waveforms from the group at $\theta=0^{\circ}$ are simple and impulsive with relatively high amplitudes. Only the stations at 1,000 or $2,000 \mathrm{~km}$ have multiple arrivals. As we rotate counterclockwise around the structure, a greater number of the synthetics in each group have multiple arrivals, and consequently, low amplitudes. Synthetics at $\theta=135^{\circ}$ and $180^{\circ}$ all have multiple arrivals. The reason for this trend is the same as in the previous modeling study. As the sources move in the direction of a line along $\theta$ peq $0^{\circ}$, a greater proportion of the elements which constitute the planar part of the grid contribute to the potentials calculated in the direction of this line. Hence, synthetics of this line become more impulsive as the source migrates from position $A$ to position $\mathrm{D}$. In contrast, the synthetics at $\theta=135^{\circ}$ and $180^{\circ}$ remain complex. The elements which contribute to these potentials are largely from the nonplanar part of the boundary. 




FIG. 10. Synthetics from the topography upwarp calculated for four source positions, five azimuths and seven distances. The topography map and cross-section with source positions are in the center. The contour interval is $1 \mathrm{~km}$. The distances, angles, and azimuths of the receivers are also shown.

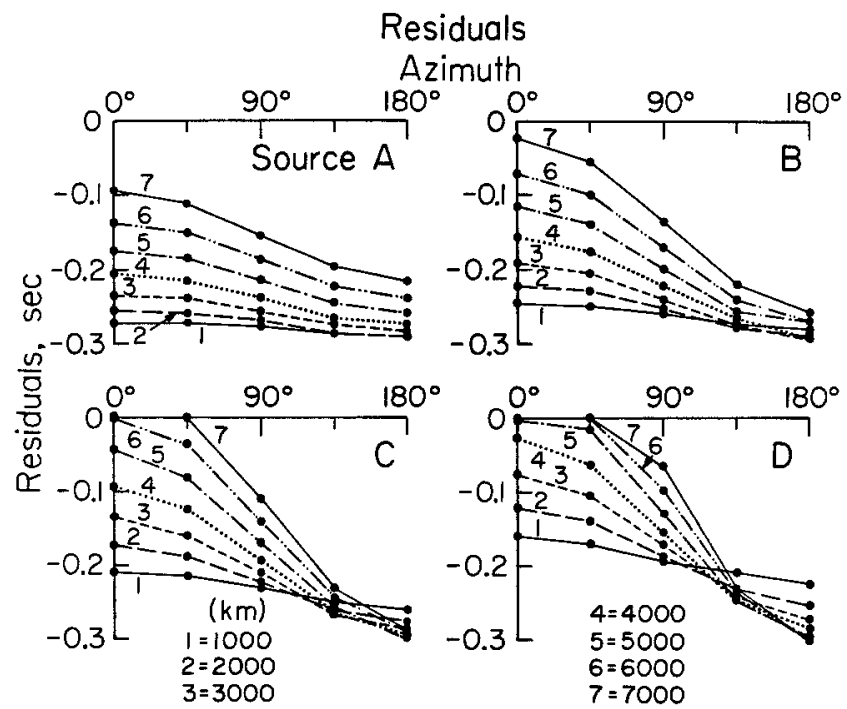

FIG. 11. Travel-time residuals for source locations A, B, C, and D plotted as a function of azimuth and distance.

We examine the maximum amplitudes, first pulse amplitudes, and travel-time residuals in Figures 11 to 13 for systematics as a function of distance or azimuth. The behavior of maximum amplitude with distance and azimuth is the most variable of the three parameters. The maximum amplitudes as a function of azimuth do not 
correlate very well with the travel-time residuals. The rapid change of this parameter with azimuth and source position reflects the sensitivity of maximum amplitudes to slight changes in relative timing between the two arrivals which make up the synthetics. The least variation of maximum amplitudes with distance and azimuth occurs at synthetics calculated with source position A, the closest source to the center of the symmetric source. We increase this variation with azimuth and distance when we remove the source further away from the center to position B, C, and $\mathrm{D}$.

The maximum amplitudes at $\theta=135^{\circ}$ for source position $\mathrm{B}, \mathrm{C}$, and $\mathrm{D}$ are lower than the amplitudes at other azimuths. This trend is a result of (1) the degree to

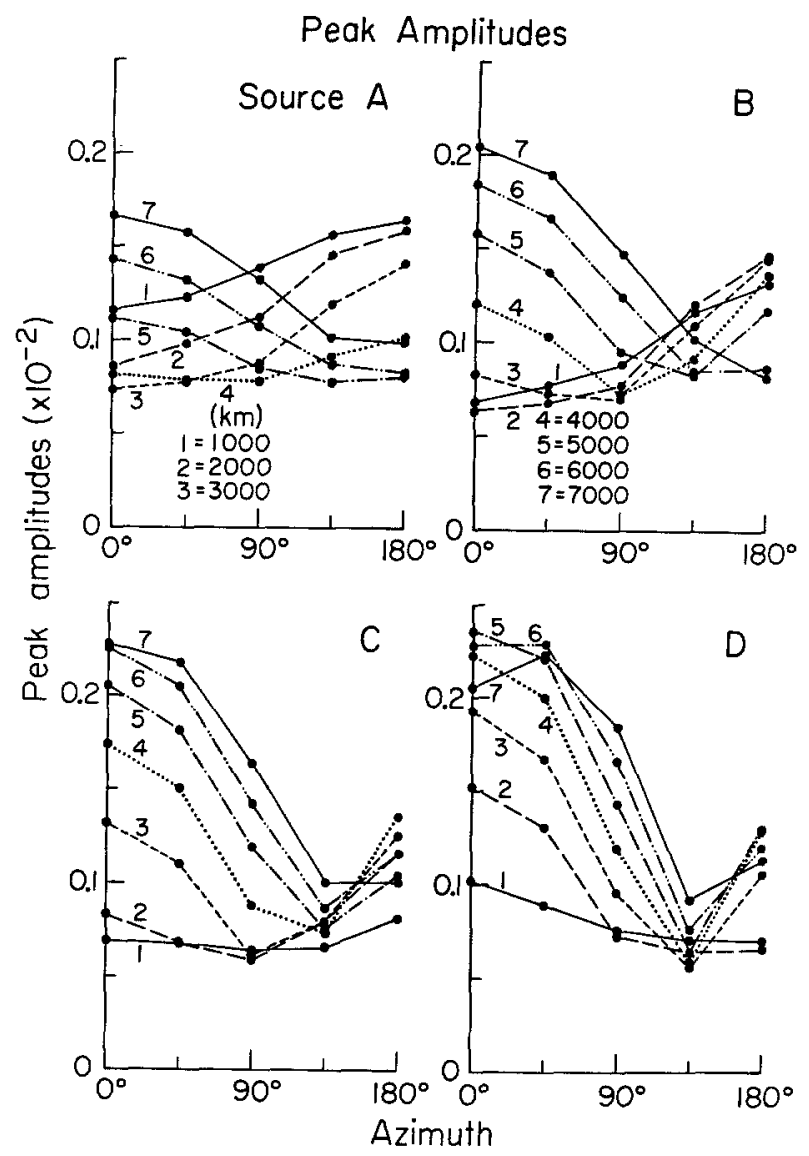

FIG. 12. Peak amplitudes for source locations A, B, C, and D plotted as a function of azimuth and distance.

which the planar part of the grid contributes to the response and (2) the degree of symmetry of the source and receiver locations with respect to the structure. Stations along $\theta=0^{\circ}$ and $\theta=180^{\circ}$ are in positions of symmetry with respect to the sources. Elements on either side of a line which divides the grid contribute simultaneously to the responses at these stations and, consequently, cause higher maximum amplitudes. Stations along $\theta=90^{\circ}$ and $\theta=45^{\circ}$ are not symmetrically positioned with respect to the source; however, the planar part of boundary largely contributes to these responses; thus, they have high maximum amplitudes. But receivers along $\theta=135^{\circ}$ are placed asymmetrically which causes elements to illuminate at different 
times; in addition, these elements are largely in the perturbed part of the boundary. These two factors combine to produce the overall lower maximum amplitudes of receivers at $\theta=135^{\circ}$.

Although we do not discern any relationship between the maximum amplitudes and the travel-time residuals as a function of azimuth, we see a correlation between the first pulse amplitudes and the travel-time residuals. Synthetics which have a constant first amplitude as a function of azimuth also have approximately constant travel-time residuals. When the travel-time advances increase as a function of azimuth, the first amplitudes decrease with azimuth. Thus, early synthetics have



FIG. 13. Amplitudes of the first pulse for source locations A, B, C, and D plotted as a function of azimuth and distance.

lower first amplitudes than the later synthetics. The trend of early arrivals with low amplitudes and late arrivals with high amplitudes holds true for all azimuths, distances, and source positions.

The travel-time residuals decrease as a function of distance at all azimuths except for $\theta=135^{\circ}$ and $\theta=180^{\circ}$ for sources $\mathrm{C}$ and $\mathrm{D}$. At these azimuths, the residuals increase as the distance increases. Clearly, if we pull the source off the center far enough, the shallower rays will interact with the upwarped part of the topography while the steeper rays interact with the flat part of the grid.

As the source moves off the center, the range of variation of first amplitudes and travel-time residuals as a function of azimuth exceeds the range of these parameters 
as a function of distance; that is, the trends of these parameters are stronger in azimuth than in distance. Thus, stronger variation of travel time and amplitude anomaly with azimuth than with distance may be an indicator of lateral variation with azimuth, as well as with distance, despite an uneven station distribution of the existing data set.

What conclusions can be drawn from this modeling experiment? First, we create a variation of $2 \frac{1}{2}$ of first amplitudes as a function of azimuth and source position. The change of amplitude with source position is largest at $0=0^{\circ}$ and $45^{\circ}$ and is the least at $\theta=180^{\circ}$. However, the variation at $0^{\circ}$ and $45^{\circ}$ is created at the cost of considerable distortion of the waveform. This feature of low amplitude waveforms with complex or broadened pulses and high amplitudes waveforms with simple narrow pulses is not apparent in the mesa data set.

Second, we create a trend in the first pulse amplitudes with azimuth. Specifically, we cause high amplitudes at $\theta=0^{\circ}, 135^{\circ}$, and $180^{\circ}$. If the source moves far enough away from upwarp, it causes no amplitude anomalies. However, structure can produce a systematic azimuthal trend in amplitudes. But we must be cautious about pushing this interpretation too far. The azimuthal trend is an artifact of the moveout of the source in one direction with respect to the lines of receivers. If we were to distribute sources all over the upwarp and then calculate the averages of the first amplitudes at each azimuth for all the sources, we would undoubtedly eliminate any trend with azimuth. Thus, the stability of the amplitude pattern of all mesa events with azimuth location is not easily explained by structure on the Moho or any unusual velocity plug unless the sources are fortuitously located to one side of the heterogeneity.

Third, we see a visual correlation between travel-time residuals and amplitudes of first pulses, but do not see any between residuals and peak amplitudes. This correlation may be diagnostic of structure as opposed to tectonic release.

\section{Discussion AND CoNCLUSIONS}

The previous two modeling experiments show that a structure on the Moho which causes travel-time residuals compatible with the Spence (1974) and the Minster et al. (1981) studies can produce variations of $2 \frac{1}{2}$ of amplitudes as a function of delta, azimuth, and source position. The variation is created at the cost of considerable distortion of the waveform. Furthermore, the travel-time residuals correlate with first pulse amplitudes but not with peak amplitudes. To see whether these initial results are relevant to the Pahute Mesa waveform data, we must now include a $p P$ phase, a $Q$ operator and a short-period instrument operator in a few Kirchhoff synthetics.

We choose two sets of five Kirchhoff-Helmholtz synthetics calculated at $4000 \mathrm{~km}$ previously for the azimuthal study. The two sets correspond to the source positions $A$ and $D$ at five azimuths. This choice represents two extremes of source positions relative to a structure and may give us a reasonable idea of what to expect in amplitude and waveform variation as test sites move within the mesa.

We put $p P$ into the Kirchhoff-Helmholtz synthetics by convolving these synthetics with a boxcar function of unit height and a width corresponding to the $p P-P$ lag time. This convolution yields the impulse response of $P$ and $p P$ if the incident source is a modified Haskell function rather than its time derivative. We justify this simple model of $p P$ by assuming that the reflection coefficient of this phase is -1 . Lay et al. (1983a) estimates the reflection coefficient as 0.96 for $p P$. We further argue that $p P$ interacts with the same part of the surface as does $P$. This assumption 
is good for the shallow depths of the mesa tests which range from 0.5 to $1.4 \mathrm{~km}$. The width of the boxcar is $0.85 \mathrm{sec}$; this estimate of the $p P-P$ lag time is taken from Lay et al. (1983). A short-period instrument and a Futterman $Q$ operator is also convolved into these synthetics. We use a Haskell function with parameters $(B=$ $2, K=10)$ while Lay et al. (1983a) use slightly different values $(B=1, K=8)$.



FIG. 14. Kirchhoff-Helmholtz responses (first column) convolved with a boxcar of width 0.85 sec and a short-period WWSSN instrument (second column) and a $Q$ operator with $t^{*}$ values of 0.5 (third column) and 1 (fourth column). Responses are from a distance of $4000 \mathrm{~km}$, azimuth range of $0^{\circ}$ to $180^{\circ}$ and source locations $A$ and $D$.

Figure 14 displays the results of the convolutions. The first column contains the initial Kirchhoff-Helmholtz synthetics with peak amplitudes taken from Figure 10. The second column show these synthetics convolved with a WWSSN short-period instrument and a boxcar. We introduce additional complexity into the waveform but do not change the range of peak amplitudes significantly when we include an 
instrument and $p P$. The complexity of the waveform caused by structure is masked by the dominant interference between $P$ and $p P$.

We next convolve these synthetics with Futterman $Q$ operators with a $t^{*}$ of 0.5 and 1 . The waveforms and their $a b$ amplitudes are displayed in columns 3 and 4 . The $a b$ amplitudes are plotted as function of azimuth for both sources and $t^{*}$ values in Figure 15. We remove the complexity of the waveform for both sources with the two $t^{*}$ values. However, there are some observable differences in the first and third peaks of the waveforms as a function of azimuth. The first peak widens as azimuth increases. The third peak becomes smaller and disappears altogether. Moreover, Figure 15 shows a variation of $a b$ amplitudes with azimuth of $2 \frac{1}{2}$ for source $D$ if $t^{*}$ is 0.5 . However, when $t^{*}$ is 1 , this variation reduces to a factor of 1.7 . We also obtain a variation of $2 \frac{1}{2}$ of $a b$ amplitudes with respect to source position if $t^{*}$ is 0.5 . This occurs at $\theta=0^{\circ}$ and $\theta=45^{\circ}$; however, the difference between $a b$ amplitudes for the two sources decreases as the azimuth increases.

Thus, we cannot predict the observed $a b$ amplitude variation with azimuth or



FIG. 15. $a b$ amplitudes from synthetics in Figure 14 plotted as a function of azimuth for both source locations $\mathrm{A}$ and $\mathrm{D}$ and both $t^{*}$ values.

station location if we use a structure on the Moho $10 \mathrm{~km}$ high and approximately $25 \mathrm{~km}$ wide. If the factors of 12 or 40 , seen in Figures 3 to 5 , are measures of a purely near-source phenomena, then one requires a structure several hundred kilometers in scale on a boundary to match these factors. This structure would distort the waveform considerably. Yet there is no obvious evidence in the observed seismograms for a correlation between low amplitudes and complicated and/or broadened waveforms or high amplitudes and simple, impulsive waveforms. We speculate that, rather than a large structure on a single boundary, a small velocity or density perturbation along a several hundred kilometer ray path may produce the desired amplitude change. However, we cannot test this speculation with our method.

On the other hand, we predict a factor of $2 \frac{1}{2}$ in $a b$ variation with source location if $t^{*}$ is 0.5 . This variation is not accompanied by any significant waveform distortion. Although we only produce a factor of $2 \frac{1}{2}$ at two azimuths, this is an artifact of the source moveout across the structure. If sources were uniformly distributed over the 
structure, we would cause this same magnitude of variation at all the azimuths. Furthermore, no source would be systematically higher in $a b$ amplitudes than another,source at all the azimuths. Unfortunately Figure 3 does not show systematics with respect to source location. The data should be examined for such trends.

Also the travel-time residuals do not correlate with the $a b$ patterns. The convolution with two $t^{*}$ values demonstrates the frequency dependence of the phenomena. If $t^{*}$ is larger than 1 , we will produce a flat pattern of $a b$ amplitudes with azimuth but the travel-time residuals will not change. Thus, we do not expect a systematic relationship between travel-time and amplitude anomalies because the $a b$ amplitudes are sensitive to $Q$ but the travel times are relatively stable. This is an unfortunate result because such a correlation would be diagnostic of structure as opposed to tectonic release. As yet, there has been no study which definitively demonstrates a relationship. In addition, the data sets of amplitude and travel-time measurements do not have a one-to-one correspondence. Lay et al. (1983a) measure the $a b$ amplitudes off of short-period WWSSN instruments while Spence (1974) and Minster et al. (1981) use culled travel-time measurements from the ISC catalogs. We clearly need a study which compares the travel time and amplitude from the same seismogram.

The largest overall variation in $a b$ amplitude with azimuth in Figure 15 occurs because of differences in $t^{*}$ values. Yet there are no noticeable changes in the waveform. Thus, the $a b$ amplitudes are far more sensitive than waveforms to $Q$ differences. Perhaps, a lateral variation of $Q$ with path can produce the extreme scatter of $a b$ amplitudes for both tests inside and outside the mesa. However, it cannot explain the differences in patterns between these different test site areas. If the near-mesa anomalies are, indeed, at least a factor of 10 , then structure on the Moho which is compatible with travel-time residuals cannot produce these large variations of amplitude with azimuth. However, such a structure could explain the observed variation of $a b$ amplitudes with source position at a given station.

\section{ACKNOWLEDGMENTS}

We wish to thank Dave Cole, Holly Eissler, Dave Harkrider, and Thorne Lay for critically reviewing this manuscript. This work was supported by ONR Contract 14-76-C-1070 and by the Air Force Office of Scientific Research under Contract F49620-83-C-0025.

\section{REFERENCES}

Aki, K. and Y.-B. Tsai (1972). Mechanism of Love-wave excitation by explosive sources, J. Geophys. Res. 77, 1452-1475.

Butler, R. (1984). Azimuth, energy, $Q$ and temperatures: variation on $P$ wave amplitudes in the United States, Rev. Geophys. Space Phys. 22, 1-36.

Haddon, R. and P. Buchen (1981). Use of the Kirchhoff's formula for body wave calculations in the Earth, Geophys. J. R. Astr. Soc. 67, 587-598.

Hilterman, F. (1975). Amplitudes of seismic waves-A quick look, Geophysics 40, 745-762.

Hong, T. L. (1978). Elastic wave propagation in irregular structures, Ph.D Thesis, California Institute of Technology, Pasadena, California.

Langston, C. (1977). The effect of planar dipping structure on source and receiver responses for constant ray parameter, Bull. Seism. Soc. Am. 67, 1029-1050.

Langston, C. and D. V. Helmberger (1975). A pronedure for modelling shallow dislocation sources, Geophys. J. R. Astr. Soc. 42, 117-130.

Lay, T., T. C. Wallace, and D. V. Helmberger (1983a). The effects of tectonic release on short-period $P$ waves from NTS explosions, Bull. Seism. Soc. Am. 74, 819-842.

Lay, T., L. J. Burdick, D. V. Helmberger, and C. G. Arvesen (1983b). Estimating seismic yield and defining distinct test sites using complete waveform information, Woodward-Clyde Report, WCCPR-84-01. 
Minster, J. B., J. M. Savino, W. L. Rodi, T. H. Jordan, and J. F. Masso (1981). Three-dimensional velocity structure of the crust and upper mantle beneath the Nevada test site, Systems Science, and Software Report, SSS-R-81-5138.

Nuttli, O. W. (1969). Travel times and amplitudes of $S$ waves from nuclear explosions in Nevada, Bull. Seism. Soc. Am. 59, 385-398.

Scott, P. and D. V. Helmberger (1983). Applications of the Kirchhoff-Helmholtz integral to problems in seismology, Geophys. J. R. Astr. Soc. 72, 237-254.

Spence, $W$. (1974). $P$-wave residual differences and inferences on an upper mantle source for the Silent Canyon volcanic centre, Southern Great Basin, Nevada, Geophys. J. R. Astr. Soc. 38, 505-523.

Stratton, J. A. (1941). Electromagnetic Theory, first edition, McGraw-Hill Book Company, Inc., New York.

Wallace, T. C., D. V. Helmberger, and G. R. Engen (1983). Evidence of tectonic release from underground nuclear explosions in long-period $P$ waves, Bull. Seism. Soc. Am. 73, 593-813.

SEISMOLOGICAL LABORATORY

Division of Geological and Planetary Sciences

CALIFORNia Institute of TECHNOLOGY

Pasadena, California 91125

ConTribution No. 4122

Manuscript received 28 June 1984 